\title{
Swallowing interventions for the treatment of dysphagia after head and neck cancer: a systematic review of behavioural strategies used to promote patient adherence to swallowing exercises
}

\author{
Roganie Govender ${ }^{1 *}$ (D), Christina H. Smith², Stuart A. Taylor ${ }^{3}$, Helen Barratt ${ }^{4}$ and Benjamin Gardner ${ }^{5}$
}

\begin{abstract}
Background: Dysphagia is a significant side-effect following treatment for head and neck cancers, yet poor adherence to swallowing exercises is frequently reported in intervention studies. Behaviour change techniques (BCTs) can be used to improve adherence, but no review to date has described the techniques or indicated which may be more associated with improved swallowing outcomes.

Methods: A systematic review was conducted to identify behavioural strategies in swallowing interventions, and to explore any relationships between these strategies and intervention effects. Randomised and quasi-randomised studies of head and neck cancer patients were included. Behavioural interventions to improve swallowing were eligible provided a valid measure of swallowing function was reported. A validated and comprehensive list of 93 discrete BCTs was used to code interventions. Analysis was conducted via a structured synthesis approach.

Results: Fifteen studies (8 randomised) were included, and 20 different BCTs were each identified in at least one intervention. The BCTs identified in almost all interventions were: instruction on how to perform the behavior, setting behavioural goals and action planning. The BCTs that occurred more frequently in effective interventions, were: practical social support, behavioural practice, self-monitoring of behaviour and credible source for example a skilled clinician delivering the intervention. The presence of identical BCTs in comparator groups may diminish effects.

Conclusions: Swallowing interventions feature multiple components that may potentially impact outcomes. This review maps the behavioural components of reported interventions and provides a method to consistently describe these components going forward. Future work may seek to test the most effective BCTs, to inform optimisation of swallowing interventions.
\end{abstract}

Keywords: Dysphagia, Head neck cancer, Swallowing exercises, Behavior change techniques, Adherence, Complex interventions

\footnotetext{
* Correspondence: Roganie.Govender@uclh.nhs.uk

'University College London, Health Behaviour Research Centre \& University

College London Hospital, Head \& Neck Cancer Centre, Ground Floor Central,

250 Euston Road, London NW1 2PQ, UK

Full list of author information is available at the end of the article
} 


\section{Background}

Swallowing difficulties (dysphagia), which affect $60-75 \%$ of patients treated for head and neck cancer (HNC) [1], arise both from the presence of a tumour, and as a consequence of its treatment [2]. Dysphagia is a major patient concern after cancer treatment due to the detrimental impact on patients' quality of life (QOL) [3]. Improvement of swallowing function and earlier restoration of eating and drinking after surgery or chemoradiation treatments may be achieved with swallowing rehabilitation exercises $[4,5]$. Despite this, nonadherence to swallowing exercises in this population is reported to be high [6].

The World Health Organization report defines patient adherence as "the extent to which a person's behaviour corresponds with agreed recommendations from a health care provider" [7]. This report highlights that adherence is influenced by multiple factors, and that increasing adherence to treatment could have a greater impact on health than trying to improve the efficacy of the treatment to which patients are encouraged to adhere. Adopting this perspective transforms the concept of patient adherence from a peripheral marker of study quality into a concept central to the intervention. The Medical Research Council's "complex intervention" guidelines highlight that multiple components at different levels may interact to bring about desired health outcomes [8]. Effectiveness of swallowing exercise interventions are determined not just by the exercises but also the broader 'behaviours of those delivering and receiving the intervention' (p.979). Complex interventions that take place as pragmatic trials under real-world conditions [9] are influenced by context factors; how interventions are implemented (where, by whom) and how patients may respond to this (uptake/adherence) [10].

Newer paradigms in systematic reviewing such as realist reviews focus on understanding how and why interventions work in some situations and not others, rather than simply investigating whether they do or do not work [11]. Sutcliffe and colleagues [12] argue the importance of recognising and identifying the critical components of complex interventions highlighting that outcomes of complex interventions cannot be solely ascribed to the primary content, in this case swallowing exercises. Traditional systematic reviews that focus exclusively on pooling effect sizes may overlook other aspects that influence outcomes. This limits our ability to differentially examine the evidence and to gather important information that may improve future interventions.

The system in which the intervention takes place and the possible interactions that may occur can be represented as a logic model [13] (Fig. 1). Swallowing exercise interventions for patients with HNC are normally implemented by trained professionals such as speech therapists within a healthcare setting, and as part of a wider cancer care pathway. The content of the intervention tends to be focused on type, timing and intensity of different swallowing exercises. Accordingly, previous reviews have been largely concerned with these exercise parameters. Langmore and Pisegna [14] suggest that exercises such as the Shaker (head lift exercise) and Mendelsohn manoeuvre (larynx elevation exercise) have good efficacy in improving swallowing function. A general review of interventions to improve eating and drinking after HNC [15] concluded

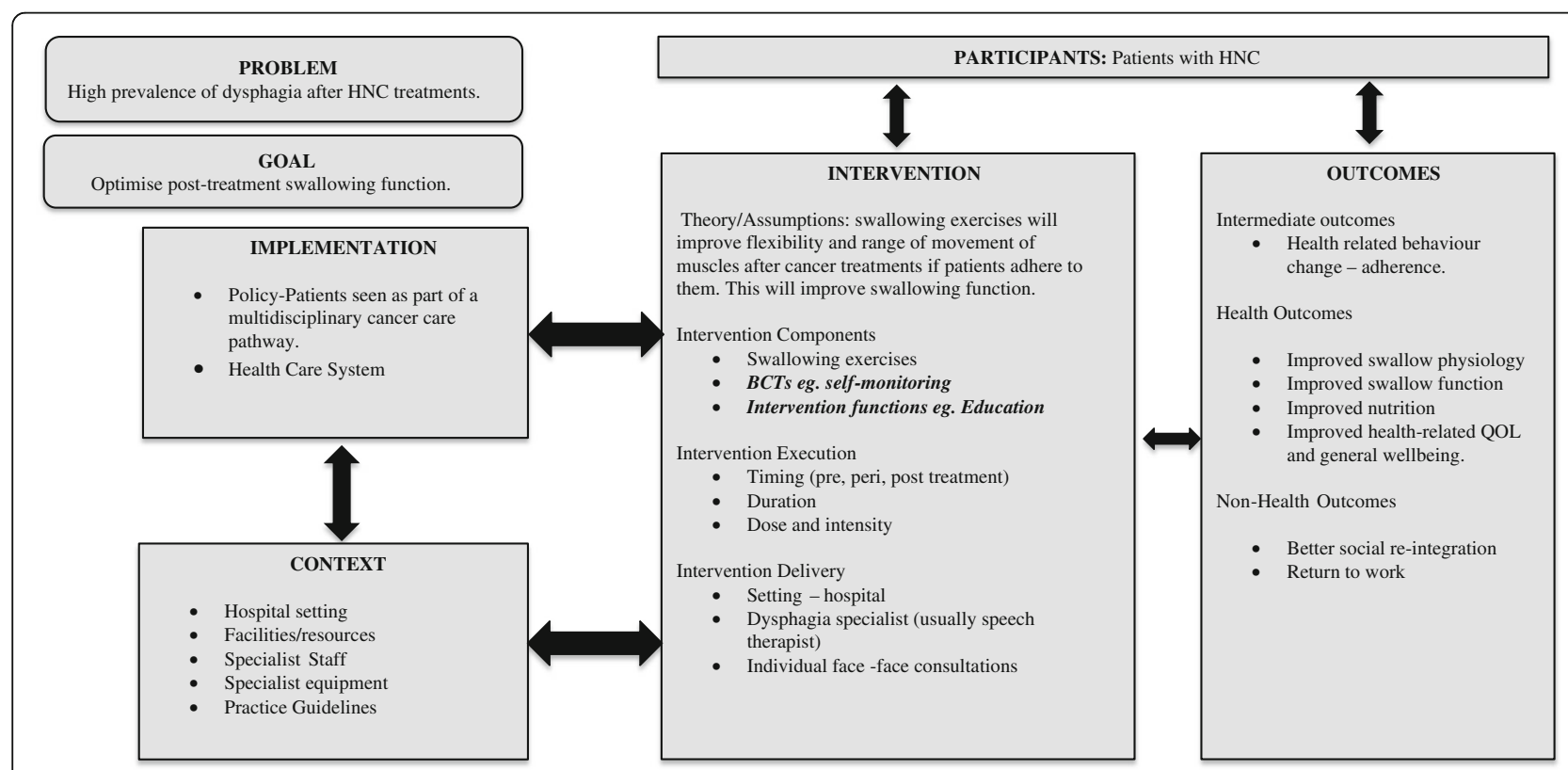

Fig. 1 Logic Model of exercise interventions to improve swallowing in patients treated for head and neck cancer 
that some evidence exists to support exercises to improve swallowing function and jaw movement in patients treated for HNC but acknowledged that larger controlled studies are needed. A recent Cochrane review [16] concluded that the evidence for pre-treatment swallowing exercises in improving swallowing safety and efficiency is lacking due to insufficiently robust studies, heterogeneity of outcome measures across studies, and poor patient adherence. Whilst there is much to be learned from these reviews, the broader perspective proposed in our logic model may facilitate better understanding of the existing evidence that could improve the content and design of future studies (Fig. 1).

As highlighted in our model, behavioural strategies used to promote adherence to the exercises are an important part of the intervention content that may be frequently overlooked yet such strategies may have a potentially crucial influence on outcomes. This review employs established tools from Behavioural Science, in particular the Behaviour Change Technique Taxonomy (BCTTv1) [17] that defines 93 discrete behaviour change techniques (BCTs) thereby facilitating a standardised description of the techniques that can be used to change behaviour. BCTs represent the smallest observable and replicable components that may bring about a change in behaviour [17], and therefore may be potentially active ingredients in an intervention [18] The success of exercise interventions is dependent on good adherence. It is logical therefore that this aspect of the intervention be given appropriate consideration.

In this review, we aim to identify the specific behaviour change strategies reported in interventions to improve swallowing function after HNC. We also explored where possible, relationships between the presence of these components and intervention effectiveness. We propose that $\mathrm{BCTs}$ that occur at least twice as frequently in successful interventions may be useful to include in future interventions. We used a narrative synthesis approach [19] and as part of this we also explored the trial methods used more broadly (for example type of comparator group), providing discussion of possible associations with the study outcomes. To our knowledge this is the first attempt to apply this method of reviewing swallowing interventions within this field, and by its nature the work is exploratory.

\section{Methods}

The review is registered with PROSPERO (CRD420 15017048), and a protocol reporting full methodological detail has been published [20].

\section{Eligibility Criteria}

Studies were eligible for inclusion where they met the following PICO criteria [21]. Participants were adults diagnosed with head and neck cancer; treated via one of the key treatment modalities of surgery, radiotherapy, chemo-radiotherapy or combinations thereof. Interventions that were eligible included behavioural interventions to improve swallowing such as swallowing exercises or instructions to adhere to a specific diet texture, and other specific swallowing strategies. Studies that included an independent comparator group were eligible - these could be randomised or non-randomised studies. The comparator group could have received no treatment (non-active comparator), usual care (active or non-active) or a different treatment (active) or sham exercise (active). For inclusion, the study had to report at least one swallow-related outcome measure which could be for example; swallow safety, swallow efficiency, swallow related QOL, oral diet intake or a surrogate marker such as feeding tube use, and textures of food tolerated. Evaluation could be via an established patient reported questionnaire, clinician rated measure or instrumental assessment tool such as videofluoroscopy.

\section{Identification of studies}

Six electronic health databases were searched: Medline, CINAHL, EMBASE, AMED, PsychInfo, and the Cochrane Library including CENTRAL. Additional searches were carried out on Google Scholar, Web of Science and the meta-registries of Trials Databases (ClinicalTrials.gov and ISRCTN). Additionally, the WHO International Clinical Trials Registry Platform (ICTRP) and the Australian New Zealand Clinical Trials Register (ANZCTR) were searched. A hand-search of reference lists of directly relevant systematic reviews and included articles identified from the main screening was also undertaken.

The search strategy was developed in conjunction with a subject librarian, following an initial scoping exercise. Medical Subject Headings from key articles and other related reviews were examined to determine the final search terms. The search was limited to clinical trials and reviews published in English. No date limit was applied.

Searches were carried out by a speech and language therapist (RG) and subject librarian (DG) in December 2014, and updated in June 2015 prior to completion of the data extraction process. One study [22] found to have two additional related reports based on longer follow-up times for the same sample and intervention, was treated as one study. Figure 2 depicts the PRISMA flowchart [23] showing the study selection process (Fig. 2).

\section{Data extraction}

\section{Study quality}

For consistency with other reviews, data was extracted on study quality using an 11-item checklist [24] used previously to assess the quality of dysphagia clinical 


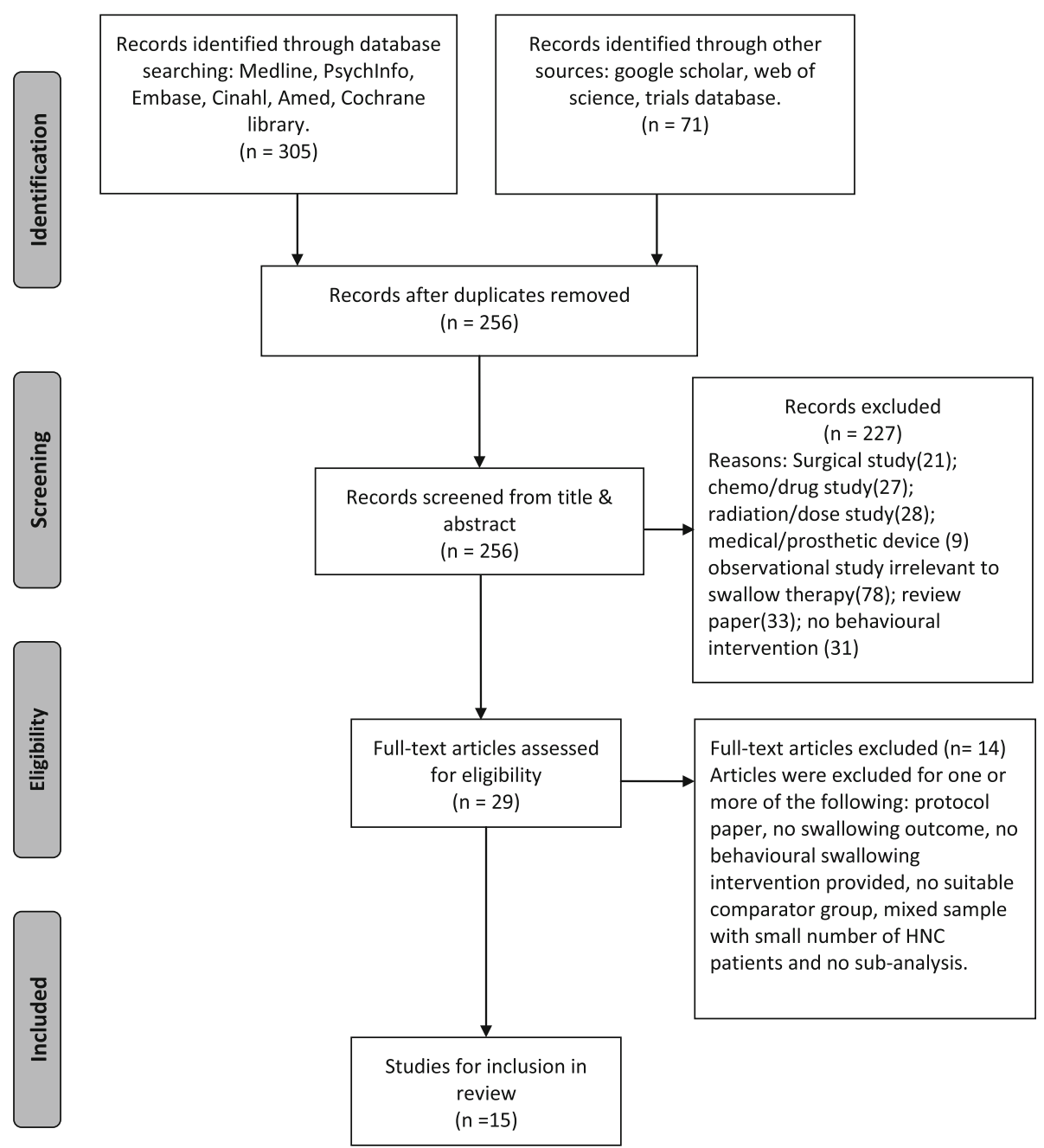

Fig. 2 PRISMA flowchart showing process of study selection

trials [25]. Each of the 11 items (Table 2) is given a score of 1 if the criterion is met, yielding a summary score of 0 (lowest) to 11 (highest quality). Van Tulder and colleagues [24] suggest that scores of $\geq 6$ reflect studies of good quality. Studies were not excluded on the basis of quality because we aimed to ascertain any evidence, however weak, of potential links between BCTs and effects. Assessing study quality and potential risk of bias is still important when synthesizing findings even if only exploratory in nature [19].

\section{Study characteristics}

Data were extracted on study characteristics (author, year, country of origin, setting, type of study), patient characteristics (diagnostic and treatment group, sample size, age range, gender and baseline swallow function), treatment (information about the type of treatment and comparator groups), and outcome measures (length of follow-up and all swallow related outcomes). We anticipated heterogeneity in the type and time-points of outcome measures but an attempt was made to extract data at or as close to the time intervals of $1,3,6$ and 12 months after treatment. They included measures derived from instrumental assessments such as modified barium swallow or videofluoroscopy, clinical measurements such as weight or the water swallow test (WST) [26], functional scales such as the Functional Oral Intake Scale (FOIS) [27] and Performance Status Scale (PSS) [28], patient-reported and QOL measures such as the MD Anderson Dysphagia Inventory (MDADI) [29] and European Organisation for Research and Treatment of Cancer (EORTC QOL C-30) [30] questionnaire.

\section{Intervention Characteristics}

For this review, we were particularly interested in identifying the behaviour change strategies (Additional file 1: Table S1 and Additional file 2: Table S2) present in the interventions. We recorded the target behaviour in each 
study, which was either regular performance of swallowing exercises or regular implementation of a prescribed diet modification with or without specific swallowing strategies. We intended to code for whether a named theory of behaviour or behaviour change was mentioned in the Abstract, Introduction, or Method, but no studies were found to have mentioned theory. We identified behaviour change strategies using BCTTv1. We also documented Intervention Function categories. Michie and colleagues [31] propose a list of nine Function categories that reflect the broad methods through which an intervention may influence behaviour: Education, Training, Enablement, Modeling, Restrictions, Environmental Restructuring, Persuasion, Incentivisation and Coercion. Both BCTs and intervention functions were only coded when they were unambiguously present in the intervention descriptions. For example if the intervention included a TheraBite device (Atos Medical, Sweden) to maintain mouth opening function - the intervention function Education was coded if it was clear that the intervention explicitly required that patients be informed and understand how the device and exercise works to maintain the ability to open the jaw. This may extend to information about the impact of radiotherapy on jaw movement and the consequences of doing/not doing the exercise. The function category Training was coded where it was clear that the patient was taught skills on how to perform the exercises using the device. The BCT demonstration on how to perform the behaviour was coded if the patient was presented with an observable demonstration, but not if only provided with written instructions; this was coded as instruction on how to perform the behaviour.

A clinician (RG) extracted data for all included studies. A speech and language therapist (CS) and health psychologist (BG) independently extracted data for four (27\%) randomly selected studies. Inter-rater agreement, assessed using Cohen's kappa, was 'substantial $\mathrm{K}=0.6$ ' or better for selection of full-text articles assessed for inclusion $(\mathrm{K}=0.86)$, study quality $(\mathrm{K}=0.74)$ and $\mathrm{BCTs}(\mathrm{K}=0.66)$ [32].

\footnotetext{
Analysis

A meta-analysis was not used due to the small number of studies and the large variability. Furthermore, it would not have been as informative for the purpose of addressing our study questions. Instead we selected a qualitative method that combined the use of summary tables, and qualitative exploration of the data.

We used a synthesis approach [19] to describe and explore our findings. Results are structured and presented in line with the key steps of this approach as listed below:
}

1. Developing a theory or model of how the intervention might work: Our logic model illustrating the interaction of various components of the intervention within a health service system has been presented above.

2. Preliminary synthesis of the findings - We summarise the characteristics of the included studies tabulating the same features across all studies. Additionally, we present summary tables of the intervention characteristics (behavioural strategies) extracted from studies and examples of these strategies obtained from content analysis of the study reports.

3. Exploring relationships in the data - We present observations of relationships between studies that may explain differences in outcomes and the direction and size of intervention effects. We assumed that BCTs that featured at least twice as frequently in studies that showed a statistically significant positive effect on at least one outcome measure $(\mathrm{p}<.05)$ in favour of the intervention group may show some promise, or at least justify more rigorous evaluation.

4. Assessing the robustness of the synthesis. We reflect on the number and quality of the studies included, and the methods used in synthesizing the findings.

\section{Results \\ Synthesis of study and intervention characteristics Study selection}

Of 374 articles identified from the combined searches, 254 remained after de-duplication. Twenty-nine articles were retained following title and abstract screening, of which 15 studies, each reporting one intervention, were eligible for review. No additional studies were included following the hand-search of reference lists.

\section{Study characteristics}

The 15 studies were undertaken across seven countries (USA, 7 studies; Netherlands and China, 2 studies respectively; Denmark, Sweden, Austria, Japan, 1 study respectively). All were carried out in a university hospital, medical centre or cancer centre. All studies sought to evaluate the impact of swallowing exercises, on one or more swallow related outcomes. Eight were randomised trials [22, 33-39], and seven were non-randomised controlled trials [40-46]. Six studies reported a comparator group of 'no treatment' $[36-38,42-44]$ and two of delayed treatment $[40,45]$. In two studies, treatment as usual was described as dietary advice without exercise [33, 34]. The comparator group for the remaining studies used a different swallowing exercise protocol described as usual care for that setting. 
Follow-ups took place between one and 12 months. The measure used for baseline swallowing status varied greatly, with 5 studies [40, 42-45] providing no report of swallowing function at baseline. At least 14 different outcome measures relating to swallow function were reported across the studies and at varied time intervals (Additional file 3: Table S3). The most frequently used measures (7/15) were: modified barium swallow and use of a feeding tube as a surrogate marker of swallow (dys) function. The PSS or a patient rated diet texture score, mouth opening, penetration-aspiration scale (PAS) [47], MDADI and weight measures were also used across multiple studies, although less frequently. Almost all studies reported a combination of instrumentally derived (objective), patient-reported and/or clinician rated outcomes measures. Two studies [42, 45] reported on just the MDADI, and one study [46] reported on a diet texture score alone.

\section{Sample characteristics}

A total of 995 participants were reported at the commencement of the studies (Table 1; 729 males, 257 females, nine unclear). Sample size ranged from 18 to 374. Average age across studies was 59.4 years. Both the gender and age demographics are broadly reflective of the epidemiology of HNC [48, 49].

Patients' HNC diagnosis ranged from stage II to stage IV disease. The sites included the oral cavity, oropharynx, hypopharynx, nasopharynx and larynx. The majority of studies (12/15), focused on the group of patients treated with radiotherapy or chemo-radiation. Of these 12 studies, ten focused on pre-treatment swallowing interventions. Three of the 15 studies [39, 42, 46] targeted patients who were treated with surgery as the main modality (Table 1 ).

\section{Quality assessment}

As indicated in Table 2, only one study [37] achieved a score $\geq 6$ and met the criteria for good quality [24]. In 7/ 15 studies, there was at least one item for which information was missing or could not be deduced from the study report. Scores ranged from $0-7$ out of 11 . No study complied with criteria requiring that the therapist and subject were blinded to the intervention (15/15) (Table 2).

\section{Intervention characteristics}

Twenty individual BCTs (Table 3) were each identified in at least one intervention. The average number of $\mathrm{BCTs}$ per intervention was seven, with a range of four to ten. The BCT instruction on how to perform the behaviour was reported in all interventions (15/15), with 14/ 15 including setting behavioural goals (for example, perform jaw exercises $3 \times /$ day) and 13/15 including action planning (for example perform exercises before mealtimes) (Additional file 1: Table S1).

A total of three Function categories were each identified in at least one intervention. Training was identified in all interventions (15/15), Education in 12/15 and Enablement for example providing patient with a TheraBite device in 5/15 (Additional file 2: Table S2).

Regular performance of the prescribed swallowing exercises was the target behaviour for all interventions. Due to the small number of studies, and the variation in exercise content we made no attempt to further group interventions according to the exercise type (Table 3 ).

\section{Exploring relationships between behavioural strategies and effectiveness}

\section{Frequency of behavioral intervention components and} intervention effectiveness

The three most commonly used BCTs that appeared in > $85 \%$ of interventions were instruction on how to perform the behaviour, setting behavioural goals and action planning. These BCTs may arguably form the cornerstone of exercise therapy interventions so it is unsurprising that they were identified in $>85 \%$ of interventions. Four BCTs were used in at least twice as many interventions that produced positive effects relative to those with no such effects - practical social support, behavioural practice/rehearsal, self-monitoring, and credible source.

\section{Exploring relationships between trial methods and effectiveness}

Influence of comparator group on intervention effectiveness We wished to explore any relations between active and non-active comparator groups and intervention effectiveness. Of five studies [22, 33-35, 43] reporting no evidence of a significantly positive effect of the intervention on any outcome, four had an active control group where similar behavioural strategies were used in both the intervention and comparator groups, except Ahlberg [43] who used parallel groups on different sites. The active comparator group represented either a different exercise regime (often described as usual care), or may have omitted the use of a swallowing exercise device that was included in the intervention group.

Of the ten interventions that demonstrated evidence of positive effects on at least one swallowing outcome measure (Additional file 3: Table S3), five [36-38, 42, $44]$ had a non-active comparator group. In two studies $[40,45]$, intervention was delayed and therefore effectively represents a non-active comparator group. Two studies had an active comparator group that received a different exercise intervention [39, 41]. One study [46] used similar exercise interventions but the intervention group included biofeedback by providing the patient with visual feedback of swallowing during a fibreoptic 


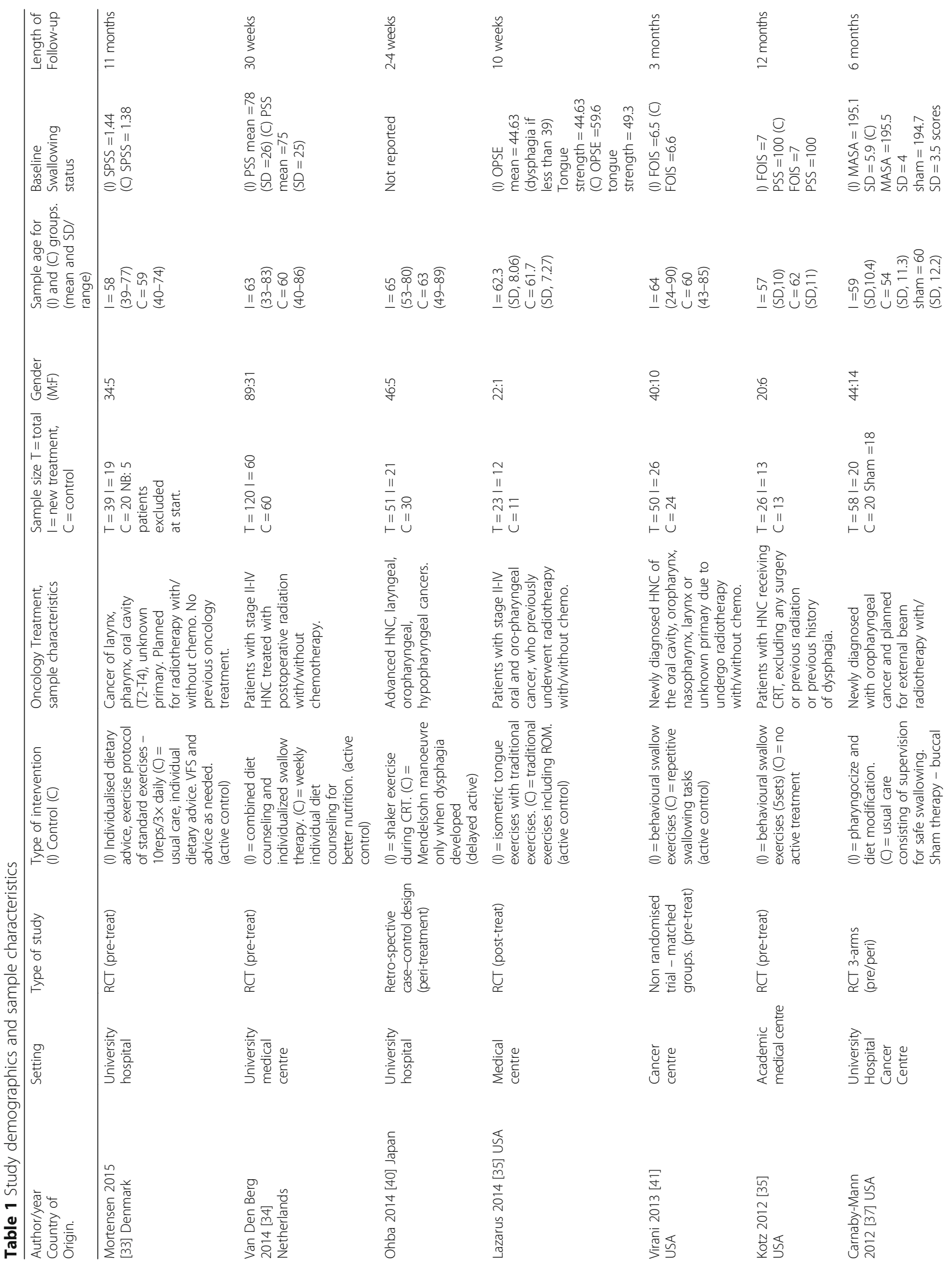




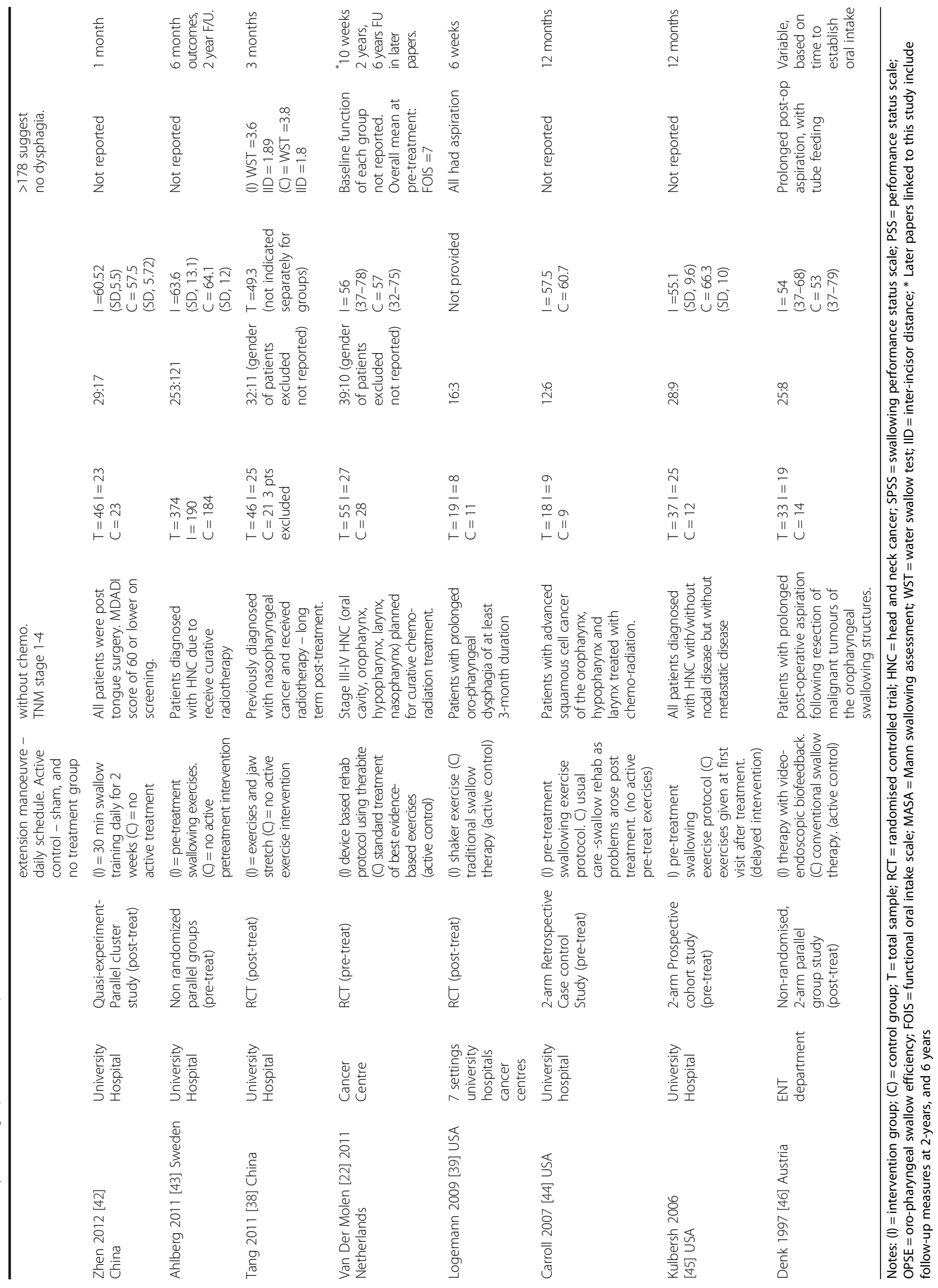


Table 2 Quality assessment ratings for all studies included in the review

\begin{tabular}{|c|c|c|c|c|c|c|c|c|c|c|c|c|c|c|c|}
\hline & Mortensen & $\begin{array}{l}\text { Van Den } \\
\text { Berg }\end{array}$ & Ohba & Lazarus & Virani & Kotz & $\begin{array}{l}\text { Carnaby } \\
\text { Mann }\end{array}$ & Zhen & Ahlberg & Tang & $\begin{array}{l}\text { Van Der } \\
\text { Molen }\end{array}$ & Logemann & Caroll & Kulbersh & Denk \\
\hline \multicolumn{16}{|l|}{$\checkmark=$ yes } \\
\hline \multicolumn{16}{|l|}{$?=$} \\
\hline \multicolumn{16}{|l|}{$x=$ no } \\
\hline \multicolumn{16}{|l|}{ Quality criteria } \\
\hline $\begin{array}{l}\text { Randomisation } \\
\text { detailed }\end{array}$ & $\checkmark$ & $\checkmark$ & $\mathrm{n} / \mathrm{a}$ & $\checkmark$ & $\mathrm{n} / \mathrm{a}$ & $?$ & $\checkmark$ & $\mathrm{n} / \mathrm{a}$ & $\mathrm{n} / \mathrm{a}$ & $?$ & $\checkmark$ & $x$ & $\mathrm{n} / \mathrm{a}$ & $\mathrm{n} / \mathrm{a}$ & $\mathrm{n} / \mathrm{a}$ \\
\hline $\begin{array}{l}\text { Allocation } \\
\text { concealed }\end{array}$ & $?$ & $x$ & $\mathrm{n} / \mathrm{a}$ & $?$ & $\mathrm{n} / \mathrm{a}$ & $x$ & $\checkmark$ & $\mathrm{n} / \mathrm{a}$ & $\mathrm{n} / \mathrm{a}$ & $x$ & $?$ & $x$ & $\mathrm{n} / \mathrm{a}$ & $\mathrm{n} / \mathrm{a}$ & $\mathrm{n} / \mathrm{a}$ \\
\hline $\begin{array}{l}\text { Similar groups } \\
\text { at baseline }\end{array}$ & $\checkmark$ & $\checkmark$ & $\checkmark$ & $\checkmark$ & $\checkmark$ & $\checkmark$ & $\checkmark$ & $\checkmark$ & $\checkmark$ & $\checkmark$ & $\checkmark$ & $\checkmark$ & $\checkmark$ & $x$ & $\checkmark$ \\
\hline Subject blind & $x$ & $x$ & $x$ & $x$ & $x$ & $x$ & $x$ & $x$ & $x$ & $x$ & $x$ & $x$ & $x$ & $x$ & $x$ \\
\hline Therapist blind & $x$ & $x$ & $x$ & $x$ & $x$ & $x$ & $x$ & $x$ & $x$ & $x$ & $x$ & $x$ & $x$ & $x$ & $x$ \\
\hline Assessor blind & $?$ & $x$ & $x$ & $\checkmark$ & $\checkmark$ & $\checkmark$ & $\checkmark$ & $x$ & $x$ & $x$ & $x$ & $\checkmark$ & $\checkmark$ & $x$ & $x$ \\
\hline $\begin{array}{l}\text { Co-intervention } \\
\text { controlled }\end{array}$ & $?$ & $x$ & $x$ & $?$ & $x$ & $?$ & $\checkmark$ & $x$ & $x$ & $x$ & $?$ & $?$ & $?$ & $x$ & $?$ \\
\hline $\begin{array}{l}\text { Acceptable } \\
\text { compliance }\end{array}$ & $x$ & $?$ & $?$ & $x$ & $x$ & $x$ & $x$ & $\checkmark$ & $?$ & $\checkmark$ & $?$ & $?$ & $?$ & $?$ & $?$ \\
\hline $\begin{array}{l}\text { Acceptable } \\
\text { withdrawal rate }\end{array}$ & $x$ & $\checkmark$ & $\checkmark$ & $x$ & $\checkmark$ & $x$ & $x$ & $\checkmark$ & $x$ & $\checkmark$ & $\checkmark$ & $x$ & $\checkmark$ & $?$ & $\checkmark$ \\
\hline $\begin{array}{l}\text { Timing of } \\
\text { outcome }\end{array}$ & $\checkmark$ & $\checkmark$ & $\checkmark$ & $\checkmark$ & $\checkmark$ & $\checkmark$ & $\checkmark$ & $x$ & $\checkmark$ & $\checkmark$ & $\checkmark$ & $\checkmark$ & $\checkmark$ & $x$ & $x$ \\
\hline $\begin{array}{l}\text { Intention to } \\
\text { treat }\end{array}$ & $\checkmark$ & $\checkmark$ & $x$ & $?$ & $\checkmark$ & $\checkmark$ & $\checkmark$ & $x$ & $x$ & $x$ & $\checkmark$ & $\checkmark$ & $x$ & $x$ & $x$ \\
\hline TOTAL & 4 & 5 & 3 & 4 & 5 & 4 & 7 & 3 & 2 & 4 & 5 & 4 & 4 & 0 & 2 \\
\hline
\end{tabular}

endoscopic assessment. One study [37] had 3 groups: a treatment group receiving swallowing exercises, a group receiving sham exercises using a similar dose schedule and a usual care group who received only safe-feeding advice by the hospital team when required but not an exercise intervention. The authors found a statistically significant difference between each of the active groups (swallowing exercises and sham exercises) and the usual care group, but a smaller difference (favouring the exercise group) between the swallowing exercise group vs sham exercise group.

Again we acknowledge the small number of studies, however our findings seem to indicate that employing active comparator groups particularly when similar behavioural strategies are used, are less likely to demonstrate statistically significant positive effects. Interestingly, a positive effect was still found in one study [46] when both groups received similar exercise interventions, but different non-exercise content (intervention group received biofeedback, a named BCT).

\section{Type and timing of outcome measures and intervention effectiveness}

Outcomes that significantly improved with the exercise intervention did so mostly at 1 month post oncological treatment, with a general decline in effect at the later time-points after treatment. Four studies measured outcomes at 12 months [33, 36, 44, 45] but only one [45] showed a significant difference in favour of the intervention by this time-point. In one study [36], outcomes were measured at multiple time-points; significant differences were observed at 3 and 6 months post-treatment but not at 9 and 12 months (Additional file 3: Table S3). Another study [33] charted a rapid decline in patient adherence to swallowing exercises over the first 12 months following treatment.

Outcomes broadly classified as objective measures (PAS, MBS score, mouth opening, feeding tube) were more frequently improved by the intervention, when compared to patient reported and clinician rated measures.

This exploration of the data has highlighted the potential impact that BCTs and trial methods such as choice of comparator group and timing of outcome measures may have on intervention effectiveness. Implications of these findings are expanded upon in the Discussion.

\section{Discussion}

We identified 15 controlled clinical trials (8 randomised) that currently represent the best available evidence of 


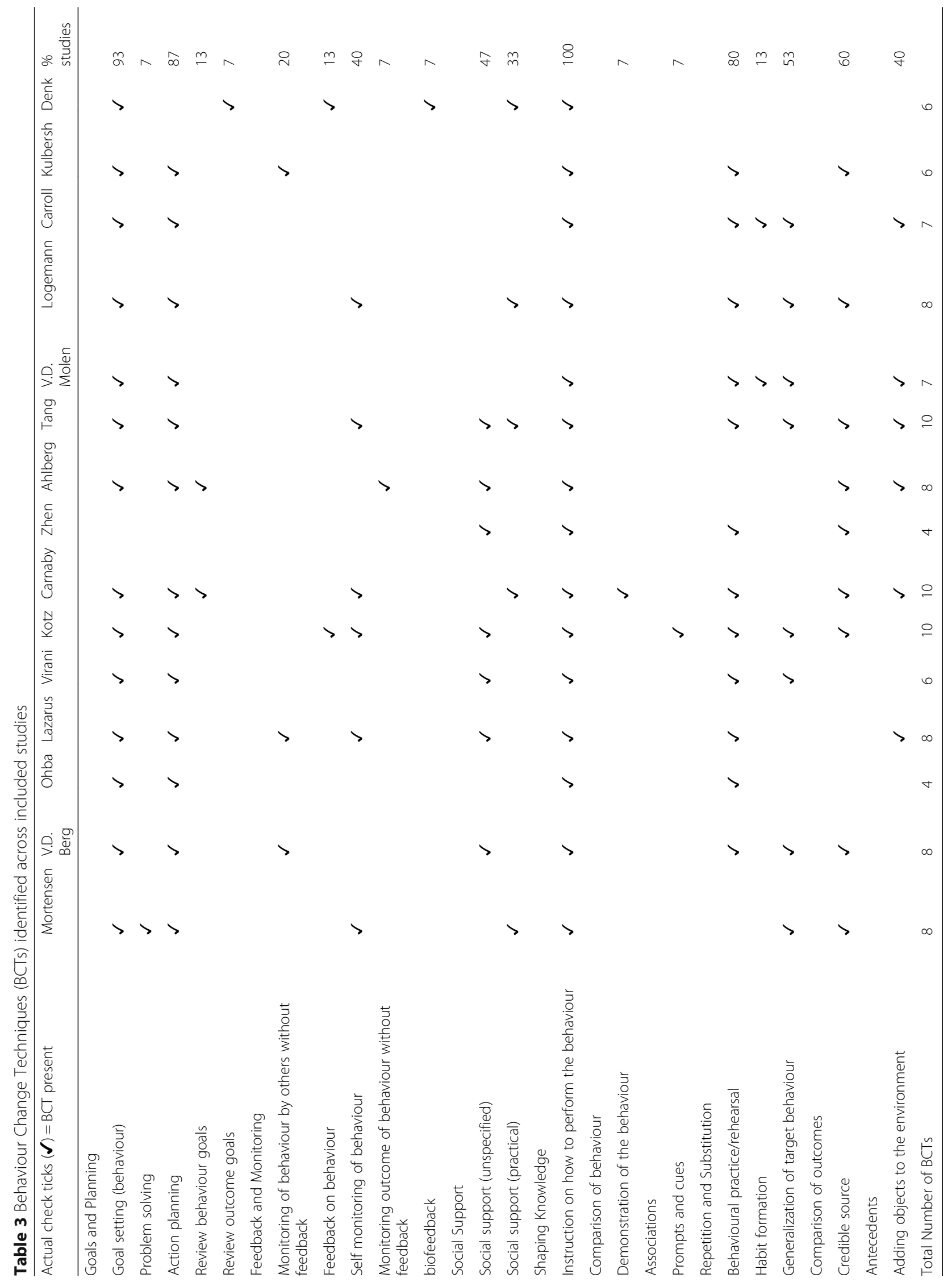


swallowing interventions for patients with $\mathrm{HNC}$, and extracted three function categories and 20 different BCTs that characterize these interventions. By specifically isolating these $\mathrm{BCT}$ s, we may encourage more consistent descriptions of the non-exercise content of swallowing exercise interventions in the literature increasing our ability to replicate studies more accurately. Indeed, in time it may be possible to devise interventions that test the effectiveness of specific BCTs or groups of BCTs used in swallowing exercise interventions for this patient population, and to link these to underlying theory and mechanisms of change [50]. In so doing, we may be better placed to understand why interventions work, for whom and in which contexts [11].

We also examined the data for any relationships that may elucidate the interaction of different components of this complex intervention. For example, studies that employed active comparator groups using similar BCTs to the intervention group were more likely to demonstrate non-significant results. Furthermore in a trial that employed three groups [37], (an exercise group, a sham exercise group, and a non-active control group), the authors reported that the active sham exercise group that received similar $\mathrm{BCTs}$ to the pharyngocize (exercise treatment) group achieved much better outcomes compared to the non-active control group. It may therefore be the constituent BCTs that were responsible for intervention effectiveness, by stimulating greater adherence to the prescribed treatment. Whilst the authors themselves did not specifically make reference to BCTs, they did question whether the "benefits obtained from the sham group could be ascribed to the placebo effect of behavioral attention' (p.219). Equally they speculated that the sham exercise (done diligently) might have had an intrinsic benefit from the increased movement of oral musculature. Regardless, these findings raise the possibility that BCTs may be functioning as active ingredients influencing intervention outcomes. For most studies where both the intervention and active comparison group used similar BCTs, no statistical significance in outcomes between groups was reported. This might be because the interventions given to both groups were too similar, or because of a lack of power due to small sample sizes. However it does raise other interesting questions: What contribution do BCTs add to intervention outcomes, and how does their presence in usual care/ placebo interventions impact effectiveness? Reporting of swallowing exercise interventions tends to focus mainly on the treatment group and often provides only cursory reference to the usual care group. The findings of this review highlight that the same methodological care should be taken in devising the treatment manuals for the intervention and comparator groups ensuring that behaviour change components are also specified, given their potential to impact patient adherence and subsequent outcomes. This may prevent hasty conclusions that imply swallowing exercises have no benefit, rather than the conclusion that the "new intervention" was not shown to demonstrate any significant additional benefit over usual care.

The variability in the type and time-point of the primary outcome measures for clinical trials in this field restricts the ability to satisfactorily pool data or compute effect sizes to address the efficacy of swallowing interventions in patients with head and neck cancer. We generally observed that in studies that reported a positive outcome, this was mostly seen in the short term. One reason for this may be because patients do not continue with their exercises long term. Behavioural strategies such as habit formation, requires that an individual repeatedly perform the behaviour in the same context such that it becomes automatic. This automaticity may promote maintenance of exercises as it may over-ride conscious intentions [51] and could have a role to play in improving swallowing outcomes longer term. We also observed that outcomes collected after 6 months showed little difference between groups. This was especially relevant for patient-reported outcomes that arguably may also reflect patients' changing expectations and adaptation over time, and not just functional swallowing status. Furthermore, this mirrors the usual trajectory of behaviour change where short-term goals are given priority. Rothman [52] highlighted that the psychological factors that underpin initiation of a new behaviour differ from those that predict maintenance of the behaviour. By implication, different BCTs may be required for these distinct phases. It was also noted that few studies actually collected objective measures of swallowing in the longer term, making it difficult to assess changes in swallow physiology at later time-points. Standardizing outcome measures and agreement on the key evaluation timepoints will greatly progress efforts to understand if swallowing exercise interventions are indeed beneficial for this group of patients and over what time period. Consideration should also be given to the expected trajectory of swallowing recovery after head and neck cancer treatment including the possible onset of late effects of treatment such as post radiation fibrosis known to impair swallow function $[53,54]$.

\section{Assessing the robustness of the synthesis}

According to Popay and colleagues [19], robustness of a synthesis is usually determined by 1 ) the methodological quality of the included studies, 2) methods used to minimise bias in the synthesis process, and 3) whether detailed information has been provided on the type of studies included/excluded. This review meets the latter two criteria by providing detailed information via a 
published protocol. Methodological quality of the available evidence was rated as poor with only one study meeting more than $50 \%$ of the applied quality criteria. It is however acknowledged that for this type of intervention, it is usually impossible to blind the therapist and subject to the intervention. Attrition is a common feature for studies that involve a complex intervention within a multifaceted cancer care pathway, and randomised studies within this field are only beginning to emerge [16, 25]. Excluding studies that did not meet quality criteria may therefore have disadvantaged our ability to address our primary aims in this exercise. Furthermore, complex interventions may require a differing emphasis on the markers of study quality as they are frequently evaluated within the context of pragmatic clinical trials. Since developing our protocol, new methods of evaluating quality in complex interventions have begun to emerge that may be more suitable for future use [9].

\section{Limitations and challenges}

This review is limited by the fact that the accuracy of the coding scheme relies on the quality of published intervention reports, which are often not sufficiently detailed to extract all necessary components of the intervention [55]. It is possible therefore that the intervention itself may have included strategies that have not been coded in this review. Descriptions of the treatment delivered to comparator groups in particular were poor, and in some cases decisions about the presence of BCTs in the comparator group had to be based on the authors' implicit suggestions that interventions were identical apart from the specific exercise protocol used in each of the active groups.

Despite the BCT taxonomy being developed within Behavioural Science, there is ongoing debate amongst experts in behaviour change as to its merits. Critics have questioned the value of coding BCTs, suggesting it creates a level of abstraction that detracts from the detailed content analysis of interventions [56]. As a counter argument, we believe that in a clinical field that has focused mainly on exercise protocol content, drawing attention to broader more abstract process based mechanisms can only enrich our understanding of complex interventions. The taxonomy brings structure, organization and a common language to this process. For example, coding a BCT such as self-monitoring may not tell us how the self-monitoring was done, but it does highlight that the use of self-monitoring may be relevant to changing adherence behaviour, particularly when it is frequently observed in successful interventions.

\section{What this review adds}

This review applied a behavior change perspective to studies within head and neck cancer swallowing rehabilitation, with a specific focus on identifying the behavioral strategies that may impact patient adherence to exercises, and consequently swallowing outcomes. Such an analysis is absent in the current literature. Our aim was to instigate discussion and greater thought about the complexity of swallowing exercise interventions, their design and the reporting of such interventions. It addresses the question of what might bring about change by isolating the specific components within an intervention, other than the nature of the treatments to which patients are encouraged to adhere, that may influence behaviour [57]. It therefore expands on the findings from previous related reviews $[15$, $16,58,59$ ] and goes some way to highlighting additional components that may be present and active in this complex intervention. Given the relative paucity of high quality data, the review did not attempt to definitively answer the question of which BCTs are most effective in promoting adherence, but instead aimed to highlight those that were prevalent in successful interventions. Using this as a starting point, we may begin to design future interventions incorporating specific BCTs or groups of BCTs to examine more closely whether they strengthen interventions aimed at improving swallowing function via swallowing exercises. Clearly BCTs are only one part of trial design and equal attention should be placed on other important aspects such as precise definition of the whole intervention package in prospective study protocols and intervention manuals.

This approach seeks to generate new discussion toward understanding the make-up of complex interventions. It also offers new perspectives in the interpretation of findings from clinical trials of swallowing exercises where it is clear that evaluating effectiveness is hampered by poor adherence.

\section{Conclusion}

The effectiveness of swallowing exercises depends in part on adherence to exercises. This review looks at $\mathrm{BCTs}$ - these seem to promote adherence. The review has provided preliminary information about which BCTs occur in reports of complex swallowing interventions and has highlighted that behavioural components may be active ingredients of change that impact intervention outcomes. It is likely that many BCTs are used in clinical practice, and there will be some bias towards the techniques that researchers tend to report. Nevertheless, introducing the taxonomy of BCTs helps equip dysphagia researchers with the tools and the language to improve consistency in how complex interventions are specified in research protocols, intervention manuals and the published literature study. In time, the approach can also be used in examining fidelity in the delivery of interventions through field testing and observational methods. Its merits and weaknesses can only be 
adequately evaluated as the body of work adopting this approach increases.

\section{Additional files}

Additional file 1: Table S1. BCTs, descriptions and examples from studies included in the review. NB: A complete list of BCTs in the taxonomy (BCTTV1) and a full description can be found in Michie, Atkins \& West [60]. (DOCX $125 \mathrm{~kb})$

Additional file 2: Table S2. Intervention Function definitions and examples from included studies where identified. NB: Further general examples of Intervention Functions can be found in Michie, Atkins \& West [60]. (DOCX $82 \mathrm{~kb})$

Addtional file 3: Table S3. Outcome measures obtained at four time points post oncology treatment. (DOCX $1082 \mathrm{~kb}$ )

\section{Abbreviations}

BCT: Behaviour change technique; BCTTV1: Behaviour change technique taxonomy Version 1; EORTC: European Organisation of Research and Treatment of Cancer; FOIS: Functional oral intake scale; HNC: Head and neck cancer; MBS: Modified barium swallow; MDADI: MD Anderson Dysphagia Inventory; PAS: Penetration-aspiration scale; PRISMA: Preferred Reporting of Items for Systematic reviews and Meta Analyses; PSS: Performance status scale; QOL: Quality of life; WST: Water swallow test

\section{Acknowledgements}

The authors wish to thank Daphne Grey for her assistance with the Database searches. We also thank Professors *Jane Wardle and Charles Abraham for their useful discussions and debate during the planning of this review.* Jane Wardle passed away on 20 October 2015

\section{Funding}

"This report is independent research supported by the National Institute for Health Research. (NIHR/HEE Clinical Doctoral Research Fellowship, Miss Roganie Govender, CDRF- 2013-04-020). Helen Barratt is supported by the NIHR Collaboration for Leadership in Applied Health Research and Care (CLAHRC) North Thames at Bart's Health NHS Trust. Stuart Taylor is a NIHR senior investigator supported by the UCLH Biomedical Research Centre. Department of Health Disclaimer: The views expressed are those of the author (s) and not necessarily those of the NHS, the NIHR or the Department of Health.

\section{Availability of data and materials}

All data generated or analysed during this study are included in this published article and its supplementary information files.

\section{Authors' contributions}

RG and BG conceived and designed the study. CS and ST contributed to further refinement of the protocol. RG and CS carried out screening of articles. RG extracted all data, CS and BG extracted a percentage of data and resolved discrepancies. RG analysed all data and drafted the manuscript- BG, $\mathrm{ST}, \mathrm{HB}, \mathrm{CS}$ provided critical feedback during iterative revisions. All authors approved of the final manuscript.

\section{Competing interests}

The authors declare that they have no competing interests.

\section{Consent for publication}

Not applicable.

\section{Ethics approval and consent to participate}

Not applicable.

\section{Author details}

${ }^{1}$ University College London, Health Behaviour Research Centre \& University College London Hospital, Head \& Neck Cancer Centre, Ground Floor Central, 250 Euston Road, London NW1 2PQ, UK. ${ }^{2}$ Division of Psychology \& Language Sciences University College London, London, UK. ${ }^{3}$ Centre for Medical

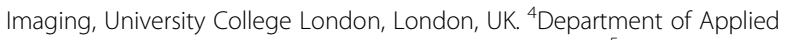
Health Research, University College London, London, UK. ${ }^{5}$ Department of Psychology, Institute of Psychiatry, Psychology and Neuroscience (IoPPN), Kings College London, London, UK \& UCL Department of Epidemiology \& Public Health, University College London, London, UK.

Received: 19 September 2016 Accepted: 9 December 2016

Published online: 10 January 2017

\section{References}

1. Malagelada JR, Bazzoli F, Boeckxstaens G, De Looze D, Fried M, Kahrilas,P et al. World Gastroenterology Organisation Global Guidelines Dysphagia Global Guidelines and Cascades Update. J. Clinical Gastroenterol;49:370-8

2. Manikantan K, Khode S, Sayed SI, Roe J, Nutting CM, Rhys-Evans P, et al. Dysphagia in head and neck cancer. Cancer Treat Rev. 2009;35:724-32. doi:10.1016/j.ctrv.2009.08.008.

3. Rogers SN, Heseltine N, Flexen J, Winstanley HR, Cole-Hawkins H, Kanatas A. Structured review of papers reporting specific functions in patients with cancer of the head and neck: 2006-2013. Br J Oral Maxillofac Surg 2016:1-7. doi:10.1016/j.bjoms.2016.02.012.

4. Wall LR, Ward EC, Cartmill B, Hill AJ. Physiological changes to the swallowing mechanism following (chemo) radiotherapy for head and neck cancer: a systematic review. Dysphagia. 2013;28:481-93. doi:10.1007/s00455013-9491-8.

5. Duarte VM, Chhetri DK, Liu YF, Erman AA, Wang MB. Swallow preservation exercises during chemoradiation therapy maintains swallow function. Otolaryngol Head Neck Surg. 2013;149:878-84. doi:10.1177/ 0194599813502310

6. Shinn EH, Basen-Engquist K, Baum G, Steen S, Bauman RF, Morrison W, et al. Adherence to preventive exercises and self-reported swallowing outcomes in post-radiation head and neck cancer patients. Head Neck. 2013;35:1707-12. doi:10.1002/hed.23255.

7. Sabate E. (ed) World Health Organization - Adherence to long term therapies. World Health Organization 2003. http://apps.who.int/iris/ bitstream/10665/42682/1/9241545992.pdf

8. Craig P, Dieppe P, Macintyre S, Mitchie S, Nazareth I, Petticrew M. Developing and evaluating complex interventions: the new medical research council guidance. BMJ. 2008;337:979-83. doi:10.1136/bmj.a1655.

9. Barratt H, Campbell M, Moore L, Zwarenstein M, Bower P. Randomised controlled trials of complex interventions and large-scale transformation of services. In Raine $R$, Fitzpatrick $R$, barratt $H$, Bevan $G$, black N, Boaden $R$, et al. Challenges, solutions and future directions in the evaluation of service innovations in health care and public health. Health Serv Deliv Res. 2016; 4(16):19-36

10. Bate P, Robert G, Fulop N, Øvretveit J, Dixon-Woods M. Perspectives on context. 2014. http://www.health.org.uk/sites/health/files/ PerspectivesOnContext fullversion.pdf.

11. Moher D, Stewart L, Shekelle P. All in the family: systematic reviews, rapid reviews, scoping reviews, realist reviews, and more. Syst Rev. 2015;4:183. doi: 10.1186/s13643-015-0163-7.

12. Sutcliffe K, Thomas J, Stokes G, Hinds K, Bangpan M. Intervention component analysis (ICA): a pragmatic approach for identifying the critical features of complex interventions. Syst Rev. 2015;4:140. doi:10.1186/s13643015-0126-z.

13. Rohwer AA, Booth A, Pfadenhauer L, Brereton L, Gerhardus A, Mozygemba $K$, et al. Guidance on the use of logic models in health technology assessments of complex interventions. http://www.integrate-hta.eu/wpcontent/uploads/2016/02/Guidance-on-the-use-of-logic-models-inhealthtechnology-assessments-of-complex-interventions.pdf. Eu/downloads/2016.

14. Langmore SE, Pisegna JM. Efficacy of exercises to rehabilitate dysphagia: A critique of the literature. Int J Speech Lang Pathol. 2015.1-8. doi:10.3109/ 17549507.2015.1024171

15. Cousins N, MacAulay F, Lang H, MacGillivray S, Wells M. A systematic review of interventions for eating and drinking problems following treatment for head and neck cancer suggests a need to look beyond swallowing and trismus. Oral Oncol. 2013;49:387-400. doi:10.1016/j.oraloncology.2012.12.002.

16. Perry A, Lee SH, Cotton S, Kennedy C. Therapeutic exercises for affecting post-treatment swallowing in people treated for advanced-stage head and neck cancers. Cochrane Database Syst Rev Published Online First. 2016. doi:10.1002/14651858.CD011112. 
17. Michie S, Richardson M, Johnston M, Abraham C, Francis J, Hardeman W, et al. The behavior change technique taxonomy (v1) of 93 hierarchically clustered techniques: building an international consensus for the reporting of behavior change interventions. Ann Behav Med. 2013;46:81-95. doi:10. 1007/s12160-013-9486-6.

18. Michie S, Abraham C, Eccles MP, Francis J, Hardeman W, Johnston M. Strengthening evaluation and implementation by specifying components of behaviour change interventions: a study protocol. Implement Sci. 2011;6:10. doi:10.1186/1748-5908-6-10.

19. Popay J, Roberts H, Sowden A, Petticrew M, Arai L, Rodgers M, et al. Guidance on the Conduct of Narrative Synthesis in Systematic Reviews: A Product from the ESRC Methods Programme. 2006:1-92. doi:10.13140/2.1. 1018.4643.

20. Govender R, Smith CH, Taylor SA, Grey D, Wardle J, Gardner B. Identification of behaviour change components in swallowing interventions for head and neck cancer patients: protocol for a systematic review. Syst Rev. 2015;4:89. doi:10.1186/s13643-015-0077-4.

21. Schardt C, Adams MB, Owens T, Keitz S, Fontelo P. Utilization of the PICO framework to improve searching PubMed for clinical questions. BMC Med Inform Decis Mak. 2007;7:16. doi:10.1186/1472-6947-7-16.

22. van der Molen $L$, van Rossum MA, Burkhead LM, Smeele LE, Rasch CRN, Hilgers FJM. A randomized preventive rehabilitation trial in advanced head and neck cancer patients treated with chemoradiotherapy: feasibility, compliance, and short-term effects. Dysphagia. 2011;26:155-70. doi:10.1007/ s00455-010-9288-y.

23. Liberati A, Altman DG, Tetzlaff J, Mulrow C, loannidis JP a, Clarke M, et al. Annals of Internal Medicine Academia and Clinic The PRISMA Statement for Reporting Systematic Reviews and Meta-Analyses of Studies That Evaluate Health Care Interventions: Ann Intern Med 2009;151:W65-94. doi:10.1371/ journal.pmed.1000100.

24. van Tulder M, Furlan A, Bombardier C. Updated Method Guidelines for Systematic Reviews in the Cochrane Collaboration Back Review Group 2003: 28(12):90-99.

25. Carnaby G, Madhavan A. A systematic review of randomized controlled trials in the field of dysphagia rehabilitation. Curr Phys Med Rehabil Rep. 2013;1:197-215. doi:10.1007/s40141-013-0030-1.

26. Hughes TA, Wiles CM. Clinical measurement of swallowing in health and in neurogenic dysphagia. QJM. 1996;89:109-16.

27. Crary MA, Carnaby-Mann G, Groher ME. Initial psychometric assessment of a functional oral intake scale for dysphagia in stroke patients. Arch Phys Med Rehabil. 2005;86:1516-20. doi:10.1016/j.apmr.2004.11.049.

28. List MA, Ritter-Sterr C, Lansky SB. A performance status scale for head and neck cancer patients. Cancer. 1990;66:564-9.

29. Chen AY, Frankowski R, Bishop-leone J. The Development and Validation of a Dysphagia-Specific Quality-of-Life Questionnaire for Patients With Head and Neck Cancer 2001;127:870-6.

30. Groenvold M, Klee MC, Sprangers MAG, Aaronson NK. Validation of the EORTC QLQ-C30 quality of life questionnaire through combined qualitative and quantitative assessment of patient-observer agreement. J Clin Epidemiol. 1997;50:441-50. doi:10.1016/S0895-4356(96)00428-3.

31. Michie $S$, van Stralen MM, West R. The behaviour change wheel: a new method for characterising and designing behaviour change interventions. Implement Sci. 2011;6:42. doi:10.1186/1748-5908-6-42.

32. Landis JR, Koch GG. The measurement of observer agreement for categorical data. Biometrics. 1977;33:159-74. doi:10.2307/2529310.

33. Mortensen HR, Jensen K, Aksglæde K, Lambertsen K, Eriksen E, Grau C. Prophylactic Swallowing Exercises in Head and Neck Cancer Radiotherapy. Dysphagia 2015:15-7. doi:10.1007/s00455-015-9600-y.

34. Van Den Berg MGA, Kalf JG, Hendriks JCM, Takes RP, Van Herpen CML, Wanten GJA et al. Normalcy of food intake in patients with head and neck cancer supported by combined dietary counseling and swallowing therapy: A randomised clinical trial. Head Neck 2015. doi:10.1002/hed.

35. Lazarus CL, Husaini H, Falciglia D, DeLacure M, Branski RC, Kraus D, et al. Effects of exercise on swallowing and tongue strength in patients with oral and oropharyngeal cancer treated with primary radiotherapy with or without chemotherapy. Int J Oral Maxillofac Surg. 2014;43:523-30. doi:10.1016/j.ijom.2013.10.023.

36. Kotz T, Federman AD, Kao J, Milman L, Packer S, Lopez-Prieto C, et al. Prophylactic swallowing exercises in patients with head and neck cancer undergoing chemoradiation: a randomized trial. Arch Otolaryngol Head Neck Surg. 2012;138:376-82. doi:10.1001/archoto.2012.187.
37. Carnaby-mann G, Crary MA, Schmalfuss I, Amdur R. Pharyngocise": randomized controlled trial of preventative exercises to maintain muscle structure and swallowing function during head-and-neck chemoradiotherapy. Radiat Oncol Biol. 2012;83:210-9. doi:10.1016/j.jijrobp.2011.06.1954.

38. Tang Y, Shen Q, Wang Y, Lu K, Peng Y. A randomized prospective study of rehabilitation therapy in the treatment of radiation-induced dysphagia and trismus. Strahlenther Onkol. 2011;187:39-44. doi:10.1007/s00066-010-2151-0.

39. Logemann JA, Rademaker A, Pauloski BR, Kelly A, Strangl-McBreen C, Antinoja J, et al. An RCT comparing the shaker exercise with traditional therapy: a preliminary study. Dysphagia. 2009;24:403-11. doi:10.1007/s00455009-9217-0.A.

40. Ohba S, Ph JY, Kojima M, Fujimaki M, Anzai T, Komatsu H, et al. Significant Preservation of Swallowing Function in Chemoradiotherapy for Advanced Head and Neck Cancer by Prophylactic Swallowing Exercise. Neck Head 2014doi: 10.1002/hed.23913

41. Virani A, Kunduk M, Fink DS, McWhorter AJ. Effects of 2 different swallowing exercise regimens during organ-preservation therapies for head and neck cancers on swallowing function. Head Neck 2013:1-9. doi:10.1002/hed. 23570

42. Zhen Y, Wang JG, Tao D, Wang HJ, Chen WL. Efficacy survey of swallowing function and quality of life in response to therapeutic intervention following rehabilitation treatment in dysphagic tongue cancer patients. Eur J Oncol Nurs. 2012;16:54-8. doi:10.1016/j.ejon.2011.03.002.

43. Ahlberg A, Engström T, Nikolaidis P, Gunnarsson $K$, Johansson $H$, Sharp L, et al. Early self-care rehabilitation of head and neck cancer patients. Acta Otolaryngol. 2011;131:552-61. doi:10.3109/00016489.2010.532157.

44. Carroll WR, Locher JL, Canon CL, Bohannon IA, McColloch NL, Magnuson JS. Pretreatment swallowing exercises improve swallow function after chemoradiation. Laryngoscope. 2008;118:39-43. doi:10.1097/MLG. Ob013e31815659b0.

45. Kulbersh BD, Rosenthal EL, McGrew BM, Duncan RD, McColloch NL, Carroll $W R$, et al. Pretreatment, preoperative swallowing exercises may improve dysphagia quality of life. Laryngoscope. 2006;116:883-6. doi:10.1016/S1041892X(07)70138-5.

46. Denk DM, Kaider A. Videoendoscopic biofeedback: a simple method to improve the efficacy of swallowing rehabilitation of patients after head and neck surgery. ORL. 1997;59:100-5.

47. Rosenbek JC, Robbins JA, Roecker EB, Coyle JL, Wood JL. A penetrationaspiration scale. Dysphagia. 1996;11:93-8. doi:10.1007/BF00417897.

48. Pytynia KB, Dahlstrom KR, Sturgis EM. Epidemiology of HPV-associated oropharyngeal cancer. Oral Oncol. 2014;50:380-6. doi:10.1016/j.oraloncology. 2013.12.019.

49. Simard EP, Torre LA, Jemal A. International trends in head and neck cancer incidence rates: differences by country, sex and anatomic site. Oral Oncol. 2014;50:387-403. doi:10.1016/j.oraloncology.2014.01.016.

50. Michie S, Carey RN, Johnston M, Rothman AJ, de Bruin M, Kelly MP, et al. From Theory-Inspired to Theory-Based Interventions: A Protocol for Developing and Testing a Methodology for Linking Behaviour Change Techniques to Theoretical Mechanisms of Action. Ann Behav Med 2016:112. doi:10.1007/s12160-016-9816-6.

51. Gardner B. A review and analysis of the use of "habit" in understanding, predicting and influencing health-related behaviour. Health Psychol Rev 2014:0:1-19. doi:10.1080/17437199.2013.876238.

52. Rothman AJ. Toward a theory based analysis of behavioural maintenance. Health Psychol. 2000;19:64-9.

53. Hutcheson KA, Lewin JS, Barringer DA, Lisec A, Gunn GB, Moore MWS, et al. Late dysphagia after radiotherapy-based treatment of head and neck cancer. Cancer. 2012;118:5793-9. doi:10.1002/cncr.27631.

54. King SN, Dunlap NE, Tennant PA, Pitts T. Pathophysiology of radiationinduced dysphagia in head and neck cancer. Dysphagia. 2016. doi:10.1007/ s00455-016-9710-1.

55. Abraham C, Johnson BT, de Bruin M, Luszczynska A. Enhancing reporting of behavior change intervention evaluations. J Acquir Immune Defic Syndr. 2014;66 Suppl 3:S293-9.

56. Ogden J. Celebrating variability and a call to limit systematisation : the example of the Behaviour Change Technique Taxonomy and the Behaviour Change Wheel 2016;7199. doi:10.1080/17437199.2016.1190291.

57. Petticrew M, Rehfuess E, Noyes J, Higgins JPT, Mayhew A, Pantoja T, et al. Synthesizing evidence on complex interventions: How meta-analytical, qualitative, and mixed-method approaches can contribute. J Clin Epidemiol. 2013;66:1230-43. doi:10.1016/j.jclinepi.2013.06.005. 
58. Speyer R, Baijens L, Heijnen M, Zwijnenberg I. Effects of therapy in oropharyngeal dysphagia by speech and language therapists: a systematic review. Dysphagia. 2010;25:40-65. doi:10.1007/s00455-009-9239-7.

59. Mccabe D, Ashford J, Wheeler-hegland K, Frymark T, Mullen R, Musson N, et al. Evidence-based systematic review: oropharyngeal dysphagia behavioral treatments. Part IV-impact of dysphagia treatment on individuals' postcancer treatments. J Rehabil Res Dev. 2009;46:205-14. doi:10.1682/JRRD. 2008.08.0092.

60. Michie S, Atkins L, West R. The Behaviour Change Wheel: A guide to designing interventions. Great Britain: Siverback Publishing; 2014.

Submit your next manuscript to BioMed Central and we will help you at every step:

- We accept pre-submission inquiries

- Our selector tool helps you to find the most relevant journal

- We provide round the clock customer support

- Convenient online submission

- Thorough peer review

- Inclusion in PubMed and all major indexing services

- Maximum visibility for your research

Submit your manuscript at www.biomedcentral.com/submit
Biomed Central 